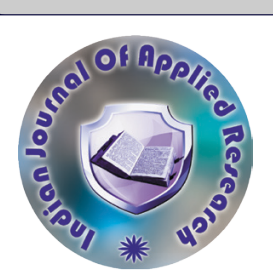

Community Medicine

\title{
RETROSPECTIVE CYTOLOGICAL STUDY OF FIBROADENOMA CASES IN A TERTIARY CARE HOSPITAL CHANDRAPUR
}

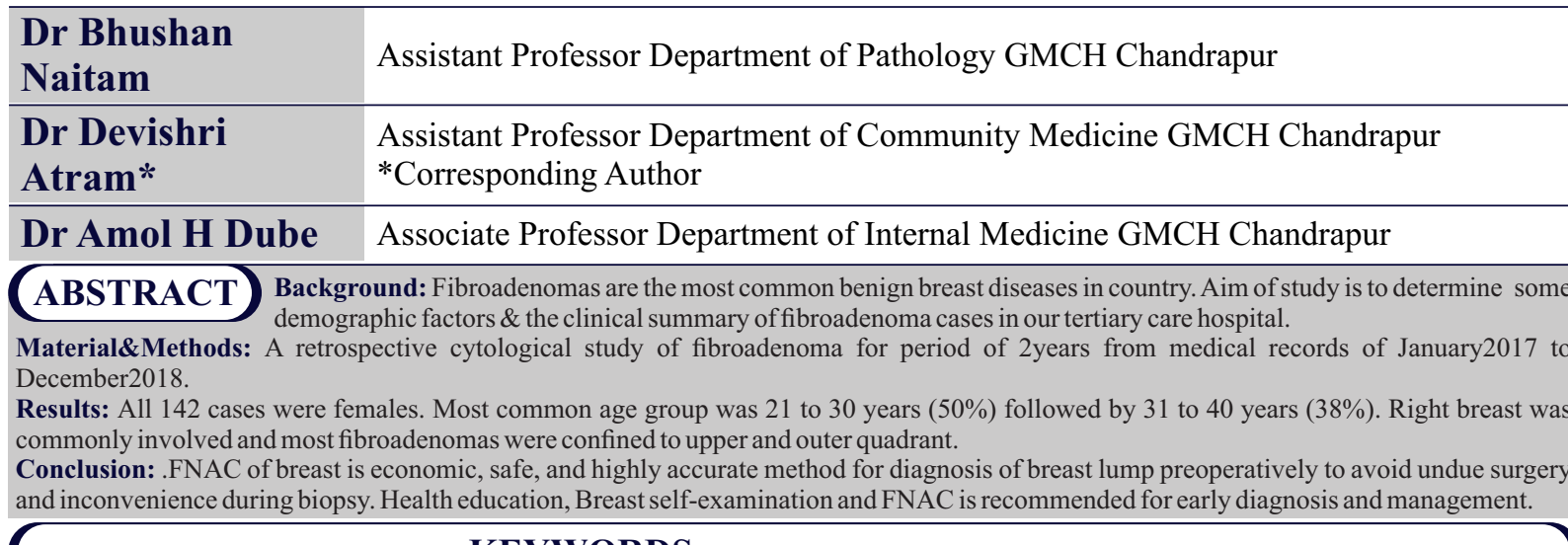

KEYWORDS : Retrospective, fibroadenoma, FNAC

\section{INTRODUCTION:}

Fibroadenomas are one of the main benign diseases of the breast characterized by an admixture of stromal and epithelial tissue. Although more common in young women, it is seen in women of any age including those who are postmenopausal. ${ }^{(1)}$ In the adolescent population, the overall incidence of fibroadenoma is $2.2 \%{ }^{(2)}$ They account for $68 \%$ of all breast masses and $44 \%-94 \%$ of biopsied breast lesions. ${ }^{(2)}$ It can rarely transform into cancerous condition also. Fine needle aspiration cytology (FNAC) and radiological imagingmammography and ultrasonography as complements to clinical examination (triple test) have become the standard approach to investigations of palpable breast lump India. ${ }^{(1,3)}$ Early diagnosis and treatment help preventing anxiety associated with nonmalignant conditions of the breast. FNAC plays a vital role in diagnosing fibroadenomas. Unlike typical lumps from breast cancer, fibroadenomas are easy to remove, with clearly defined edges. ${ }^{(3,4)}$ This study has been done to know some demographic and clinical characteristics of fibroadenoma cases which is important for early diagnosis and management.

\section{MATERIALSAND METHODS:}

It was a retrospective record based study,performed in Central pathology laboratory, Government Medical College \& Hospital, Chandrapur. Required institutional ethical committee approval was obtained for the study. Study Population included breast fibroadenoma cases from medical records January 2017 to December 2018. By using universal sampling method 142 patients were included in the study from medical records of 2 years. Reports having major typographical errors were excluded from the study. Study Procedure involves case reports having patient age and sex, history and clinical examination, breast cytology sampling technique, and conclusive breast cytology diagnosis is included in the study. Informed consent was taken from patients before sample collection. Samples from the patients were collected by using 20-22 gauge needle. Aspirated material was expressed on a clean glass slide using $20 \mathrm{cc}$ syringe. Another slide was placed on the material and spread by pulling apart to prepare a smear. The slide was immediately transferred to jar containing isopropyl alcohol. Staining was done with Haematoxylin and Eosin, Pap, May Grunwald Giemsa stain. All data was analysed using Microsoft Excel 2007 \& SPSS.

\section{RESULTS AND DISCUSSION:}

Total fibroadenoma cases from 2years duration were recorded as 142 in present study;out of which all were females. Ajitha M B et al ${ }^{(5)}$ conducted a study in Bangalore on fibroadenoma patients in which all 210 cases were females. A Mukhopadhyay et al ${ }^{(6)}$ performed a 5 year study in West Bengal on breast diseases all were female participants. S Vinoth Kumar et al (7) carried out a study in Tamil nadu in which 91\% fibroadenoma patients were females. S Durge et al (8) have done a study in Maharashtra where all fibroadenoma cases were female patients. Above mentioned study population is similar to present study.

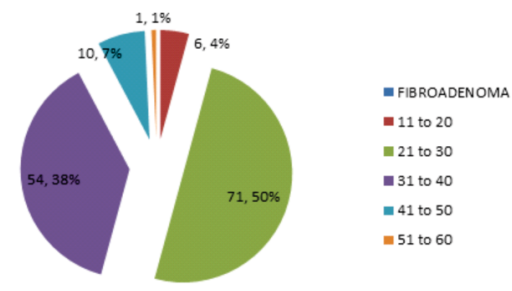

Fig1:Age group wise distribution of fibroadenoma cases

Fig 1 shows age group wise distribution of fibroadenoma cases in present study. Maximum cases $71(50 \%)$ belonged to age group 21 to 30 years followed by $54(38 \%) 31$ to 40 years and $10(7 \%)$ cases were of age group 41 to 50 years. Only $6(4 \%)$ cases belonged to 11 to 20 years and $1(1 \%)$ case belonged to 51 to 60 years age group. A Mukhopadhyay et al ${ }^{(6)}$ performed a 5 year study in West Bengal on breast diseases the mean age of benign lesion was 29 years in their study. S Vinoth Kumar et al ${ }^{(7)}$ carried out a study in Tamil nadu in which $40 \%$ of female patients were in $21-30$ years followed by $37 \%$ in 31-40 years. Abhijit MG et $\boldsymbol{a l}^{(9)}$ reported commonest age group as 2040years, and mean age as 28.6 years. S Durge et al ${ }^{(8)}$ have conducted a study in Maharashtra where commonest age group for fibroadenoma mentioned was 20-29 years. These reported study findings are consistent with present study.

\begin{tabular}{|c|c|}
\hline Side of breast fibroadenoma & Number (percentage) \\
\hline Right breast & $110(77 \%)$ \\
\hline Left breast & $22(16 \%)$ \\
\hline Bilateral & $10(7 \%)$ \\
\hline Total & $142(100 \%)$ \\
\hline
\end{tabular}

Fig:2 Side of breast involved in fibroadenoma cases

Fig:2 indicates that right breast was involved among 110(77\%) cases of fibroadenoma whereas left breast was involved among 22(66\%) cases and $10(7 \%)$ cases were bilateral involving both breasts. . Ajitha M B et al ${ }^{(5)}$ conducted a study in Bangalore on fibroadenoma patients in which all 210 cases were females out of which $103(49 \%)$ cases were involving right breast .P Rangaswamy et al ${ }^{(10)}$ conducted clinical study in Tamil Nadu on fibroadenoma in which out of 80 cases $40(50 \%)$ cases of fibroadenoma involved right breast. P Sathasivam et al ${ }^{(11)}$ conducted a study in Malaysia where only $19 \%$ of fibroadenoma cases involved right side, this is in contrast to our study 
finding. S Durge et al ${ }^{(8)}$ have conducted a study in Maharashtra where right breast (58\%) was more commonly involved for fibroadenoma cases. Brajesh Kumar et al ${ }^{(12)}$ conducted a study in Dehradun where most of bening breast diseases (52\%)were confined to right breast. Our study finding is consistent with most of the above mentioned studies.

\begin{tabular}{|c|c|}
\hline Quadrant involved & Number (percentage) \\
\hline Upper outer & $79(56 \%)$ \\
\hline Upper inner & $32(23 \%)$ \\
\hline Lower outer & $3(2 \%)$ \\
\hline Lower inner & $18(13 \%)$ \\
\hline Central & $5(3 \%)$ \\
\hline Multiple & $5(3 \%)$ \\
\hline total & $142(100 \%)$ \\
\hline
\end{tabular}

Fig:3 Breast quadrant wise distribution of fibroadenoma cases

Fig 3 dentoes maximum $79(56 \%)$ cases of fibroadenoma were confined to upper outer quadrant of breast followed by $32(23 \%)$ in upper inner quadrant.3(2\%) were in lower outer,18(13\%) were in lower inner quadrant and $5(3 \%)$ each in central and multiple quadrants. Ajitha $\mathbf{M}$ B et al ${ }^{(5)}$ conducted a study in Bangalore on fibroadenoma patients in which all 210 cases were females out of which maximum lumps 92(43\%) were in upper outer quadrant. Brajesh Kumar et al ${ }^{(12)}$ conducted a study in Dehradun where most of bening breast diseases (39\%)were confined to upper outer quadrant. S Vinoth Kumar et al ${ }^{(7)}$ carried out a study in Tamil nadu in which maximum (37\%)fibroadenomas were confined to upper outer quadrant. Sandeep Kumar Goyal et al ${ }^{(13)}$ conducted a study on breast diseases in which most of the breast lumps(34.9\%) involved upper outer quadrant. Above mentioned studies reported similar findings to present study.

\begin{tabular}{|c|c|}
\hline Consisitency of breast lump & Number(percentage) \\
\hline Firm & $136(96 \%)$ \\
\hline Soft & $6(4 \%)$ \\
\hline Hard & $0(0 \%)$ \\
\hline Total & $142(100 \%)$ \\
\hline
\end{tabular}

Fig:4 Consistency wise distribution of fibroadenoma cases

Fig 4 shows that maximum $136(96 \%)$ fibroadenoma were firm in consistency and 6(4\%) were soft in consistency and none were hard in consistency. S Durge et al ${ }^{(8)}$ have conducted a study in Maharashtra where maximum 91 (82\%) fibroadenomas were firm in consistency. $\mathbf{S}$ Sharma et al ${ }^{(14)}$ reported a case of fibroadenoma which was giant and firm in consistency. Felecia Cerrato et al ${ }^{(15)}$ mentioned in their article that most of the fibroadenomas presents with firm consistency. Most of these study finding result is consistent with present study.

\section{CONCLUSION:}

Fibroadenomas are the most common benign breast lesion with most common age group of presentation was 21 to 30 years $(50 \%)$ followed by 31 to 40 years (38\%). Right breast was commonly involved and most fibroadenomas were confined to upper and outer quadrant. FNAC serves as a rapid and economical tool for diagnosis of palpable breast lesions because of the cytopathological examination of these lesions before operation or treatment serves as an important diagnostic modality. Health education, Breast self-examination and FNAC is recommended for early diagnosis and management.

\section{REFERENCES:}

1) Saikia B, Choudhury D, Sharma I. Fine Needle Aspiration Cytology of Fibroadenoma of Breast in a Tertiary Level Hospital. Int J Sci Stud 2017;5(3):24-28.

2) Michelle Lee Hooman $T$ Soltanian.Breast fibroadenomas in adolescents: current perspectives.Dove press journal Adolescent Health, Medicine and Therapeutics 2015:(6) 59-63.

3) Berkow R. Fibroadenoma at Merck Manual of Diagnosis and Therapy. Home Edition. Whitehouse Station, NJ: Merck Research Laboratories; 1992

4) Tavasoli FA, Devilee P. World Health Organization Classification of Tumors; Pathology and Genetics; Tumors of the Breast and Female Genital Organs. Lyon: IARC Press; 2003.

5) Ajitha M B, Srinivasan N, Shivaswamy B S, Abhishek Vijayakumar. A systematic study on fibroadenoma of the breast;IJBAR (2012) 03(12): 891-5.

6) ArunimaMukhopadhyay, SuklaNaskar, Ramprasad Dey, KeyaBasu. Female Breast Lesions - A Five Year Study in a Tertiary Care Centre;International Journal of Anatomy, Radiology and Surgery. 2017 Jan, Vol-6(1): SO10-SO14.

7) Kumar SV, Kumar GN, Vinotha T, Anandan H. Breast Fibroadenomas in a Tertiary Care Hospital: A Prospective Observational Study. Int J Sci Stud 2017;4(11):176-179.

8) S Durge, A Gajbhiye, V Nasare. Characteristics of surgically treated benign breast disease. JMSCR December 2018 ; $\operatorname{vol} 6$ (12) :603-5.

9) Abhijit MG, Anantharaman D, Bhoopal S, Ramanujam R. Benign breast diseases: experience at a teaching hospital in rural India. International Journal of Research in Medical Sciences. 2017; 1(2):73-8.

10) Purushothaman R, Rubby SA. Clinical study on fibroadenoma of the breast. Int Surg J 2016;3:1916-9.
11) Priyangaa Sathasivam, Abilasha. Frequency of Various Types of Breast Lumps in Females among Malaysian Population. J. Pharm. Sci. \& Res. Vol. 7(10), 2015, 866-868.

12) Brajesh Kumar, Nitish Khandelwal, AK Paliwal, Manashi Ghosh. Clinico- radiological -pathological study of benign breast diseases. International Journal of Contemporary Medical Research 2018;5(12):L1-L4.

13) Goyal SK, Choudhary D, Beniwal S, Kapoor P, Goyal PK. Retrospective analysis of breast lumps in a given population: an institutional study. Int Surg J 2016;3:1547-50.

14) Sharma S, Rana BP. Giant fibroadenoma of breast: a diagnostic dilemma in a middle aged woman. Adv Cytol Pathol. 2017;2(4):109-112.

15) Felecia Cerrato, Brian I. Labow. Diagnosis andManagement of Fibroadenomas in the Adolescent Breast. Semin Plast Surg 2013;27:23-25. 